radiation is to make them less dependent on accurate tracking, and such designs were also reported.

lhe meeting showed clearly that the photovoltaic community in the United States is rapidly growing. The United States administration is clearly determined to make a success of photovoltaics. Indeed the considerable sum already being channelled by the US Department of Energy into this work is liable to be further increased if a Bill introduced by Congressman Mike McCormack (Democrat, Washington) is approved. This 'Solar Photovoltaic Energy Research Development and Demonstration Act of 1978' calls for the expenditure of an additional 1.5 thousand million dollars over the next ten years. This would give 125 million dollars for the fiscal year 1979. With expenditure of this magnitude many more scientists, technologists and industrial firms must be expected to apply their ingenuity to these problems.

\section{Excitation of nuclear giant resonances by heavy ions}

\section{from P. E. Hodgson}

THFRE is currently great interest in the collective modes of oscillation of highly excited nuclei. In these collective excitations, the nucleons move together in a correlated way, in contrast to the single-particle excitations which involve only one or a very few nucleons. The simplest examples of collective excitation are the vibrations of the soft nuclei and the rotations of the deformed hard nucici, and in addition therc are more energetic excitations that involve the bulk motion of the protons relative to the neutrons.

Such modes of oscillation can be excited by a projectile that exerts a strong electric field; this acts on the protons but not on the neutrons, and so the protons are all pulled away from the neutrons, and thereafter the protons and neutrons oscillate relative to each other. These excitations produce a broad maximum in the cross section of the reaction that excites them, and these are known as giant resonances. The first giant resonance to be studied was the dipolc resonance at about 22 $\mathrm{MeV}$ in light nuclei, hut since then many higher resonances have been found using a variety of projectiles and reactions. As might be expected from their collective character, the

P. E. Hodgson is a lecturer in Nuclear Physics in the University of Oxford.

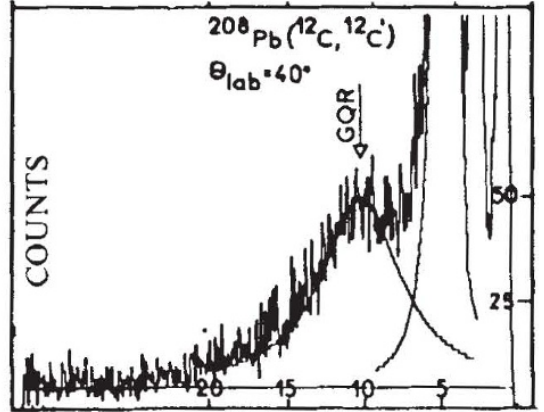

Excitation energy in Mev

Energy spectrum of ${ }^{12} \mathrm{C}$ ions inelastically scattered by ${ }^{208} \mathrm{~Pb}$ showing the peak due to the excitation of the giant quadrupole resonance.

energies and widths of the resonances change rather slowly from one nucleus to the next, as they are not dependent on the finer details of nuclear structure.

The first theories of the giant resonances used the hydrodynamical model of two fluids oscillating relative to each other, and this was able to account for the slow variation of the energy with mass number. Later on. microscopic theories were developed, and these attribute the excitation to a coherent supcrposition of all possible particle-hole excitations of the nucleons from one shell to the next. The theory is being developed and applied to the higher resonances. In particular, it has been found that they are strongly excited by proton inelastic scattering. and the cross-sections have been analysed to provide evidence for the quadrupole $(L=2)$, octupole $(L=3)$ and hexadecapole $(L=3)$ resonances in addition to the familiar dipole resonance (see Nature 246, 250; 1973). Other studics have been made using deuterons, helions and $\alpha$-particles as bombarding particles.

This work has recently been extended by Buenerd $e t$ al. of Grenoble using the heavy ions ${ }^{12} \mathrm{C}$ and ${ }^{14} \mathrm{~N}$ as projectiles to excite giant resonances in a series of nuclei from ${ }^{41} \mathrm{Ca}$ to ${ }^{209} \mathrm{Bi}$ (Phys. Rev. Lett. 40. 1482: 1978). Heavy ion reactions have the advantage that they can transfer a large amount of orbital angular momentum to the target nucleus, and thus cxcite resonances that cannot be excited by lighter projectiles. It is also to be expected that some competing processes such as quasi-free and pre-compound emission are unlikely in heavy ion reactions, so that the background is less and the giant resonances are correspondingly more prominent.

In the now experiments, each nucleus was studied by measuring the energy distribution of the inelastically scattered ions near the grazing angle, to maximise the probatility of high momentum transfer. One of these spectra is shown in the figure, and it is apparent that the peak due to the giant resonance is clearly resolved. In the case of the resonance excited by ${ }^{14} \mathrm{~N}$ on ${ }^{20} \mathrm{~Pb}$ the cross section was measured at several angles and a distorted wave calculation with $L=2$ and a deformation parameter of 0.09 was found to fit the data well, thus confirming the assignment to the giant quadrupole resonance. The integrated cross section corresponds to about $90 \%$ of the energy-weighted sum-ruie limit for E:2 transitions, and agrees with the results of other determinations.

These experiments show that heary ions can excite the giant resonances in a wide range of nuclei with good intensity relative to the background. Comparison with the results obtained using lighter projectiles sometimes show differences, and these could be due to the presence of higher multipolarities in the heavy ion reactions. and further work could lead to their identification and analysis. This work establishes heavy ions as powerful probes of highly-cxcited collective nuclear sites, and further studies using this method should lead to increased knowledge of them.

\section{Rising magma}

\section{from Peter J. Smith}

FEw would now dispute the view that much, if not all, of the volcanic material reaching the Earth's surface originates in the upper mantle. And most Earth scientists would probably agree that magma results largely from the pressure release melting of mantle rock in the rising limbs of convection cells. In the ascending solid state current beneath an oceanic ridge, for example, partial melting of mantle rock occurs, the partial melt fraction becoming the basalt of the new oceanic crust and the residue becoming part of the lithosphere below the Moho. General statements such as these arc deceptive, however, implying a degrec of knowledge that simply does not exist. The truth is that the mechanism by which magma is transported from the asthenosphere to the Earth's surface is poorly understood, whether the magma rises beneath occanic ridges, contributes to back-arc volcanism or leads to intraplate eruption.

As far as flow through the lithosphere is concerned, the simplest approach is to assume that a channel exists for the magma; but that begs the question of how the channel first 